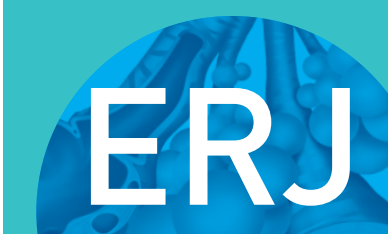

open research

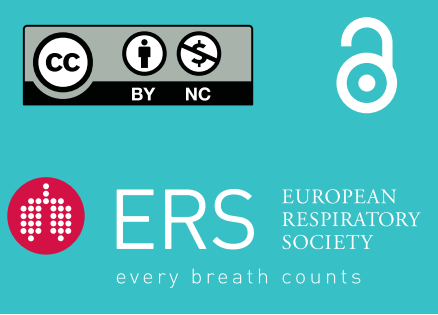

\section{Pembrolizumab-induced pneumonitis}

To the Editor:

Immune checkpoint inhibitors are promising new therapies for advanced cancers. In particular, antibodies against programmed cell death 1 (PD-1), such as nivolumab and pembrolizumab, increase anti-tumour T cell responses by blocking the interaction between PD-1 on T cells and its ligand PD-L1 on cancer cells [1]. Enhanced T-cell responses resulting from checkpoint inhibition can lead to an unusual spectrum of side effects called immune-related adverse events, which involve numerous organs, particularly the skin, liver, and gastrointestinal and endocrine systems [2-11]. Pneumonitis is a potentially lethal side effect of immune checkpoint inhibition, occurring in $1-5 \%$ of patients enrolled in trials [2-11]. However, little is known about the clinical and radiological features of checkpoint inhibitor-induced lung disease. Here, we report three cases of pembrolizumab-induced acute interstitial lung disease (ILD). The medical data of the patients presented below were collected in accordance with French regulations, none of which was opposed to their uses.

The first patient was an 82-year-old man enrolled in a clinical trial of pembrolizumab $\left(10 \mathrm{mg}^{-1} \cdot \mathrm{kg}^{-1}\right.$ every 3 weeks) for treatment of pulmonary metastatic melanoma diagnosed 2 years before. He was previously treated by surgery and temozolomide (Temodal). A pre-treatment computed tomography (CT) scan showed many secondary pulmonary nodules (figure 1a). After 11 pembrolizumab infusions, the patient presented with acute dyspnoea, cough, and sputum production associated with bilateral basal crackles. Results from standard and immunologic laboratory tests were normal. Results of pulmonary function tests were normal and no oxygen desaturation was found on the 6-min walk test. A CT scan showed bilateral areas of consolidations with ground-glass opacities (figure 1b). Bronchoalveolar lavage fluid (BALF) analysis showed a cell count of $6.5 \times 10^{5}$ cells $\cdot \mathrm{mm}^{-3}$ with $22 \%$ lymphocytes and $15 \%$ neutrophils. Results on microbiological testing of BALF were negative (including staining and culture for bacteria, fungi, viruses, mycobacteria and Pneumocystis jirovecii). The patient was treated with empiric antibiotics and a repeat CT scan revealed a migration of consolidations and ground-glass infiltrates (figure 1c). Thus, clinical and radiological features were consistent with organising pneumonia (OP) induced by pembrolizumab treatment. In accordance with the clinical study design, pembrolizumab treatment was stopped. Oral prednisolone was started at $0.5 \mathrm{mg}^{-1} \cdot \mathrm{kg}^{-1} \cdot \mathrm{day}^{-1}$ for 20 weeks (initial dose: $40 \mathrm{mg} \cdot \mathrm{day}^{-1}$ ) and then slowly tapered over 32 weeks (total 12 months of treatment; the dose was gradually decreased by $10 \mathrm{mg}$ per $10 \mathrm{mg}$ every 3 weeks until reaching a dose of $20 \mathrm{mg}$. A more mild decrease of $2.5 \mathrm{mg}$ per $2.5 \mathrm{mg}$ every month was then initiated until treatment was stopped). Three months after starting corticosteroids, the patient's clinical condition improved, with decreased dyspnoea and a marked regression of consolidation on CT scan (figure 1d). The improvement continued over the following year and the patient has remained progression-free from melanoma for 20 months without reintroduction of any therapy.

The second case was an 82-year-old man with metastatic melanoma (parotid localisation and lung metastasis) who was started on pembrolizumab $\left(2 \mathrm{mg}^{-1} \cdot \mathrm{kg}^{-1}\right.$ every 2 weeks) as second-line therapy (first-line therapy comprised surgery and radiotherapy). A CT scan performed before pembrolizumab treatment showed many bilateral pulmonary nodules (figure 1e). After four infusions, the patient reported progressively worsening dyspnoea and cough without hyperthermia. A chest CT scan showed an abundant left-sided pleural effusion with multiple confluent pulmonary consolidations prominent in the upper lobes (figure 1f). Thoracocentesis revealed a transudate (protein $15 \mathrm{~g} \cdot \mathrm{L}^{-1}$, lactate dehydrogenase $118 \mathrm{UI} \cdot \mathrm{L}^{-1}$ ) without malignant cells. BALF contained $2.8 \times 10^{5}$ cells $\cdot \mathrm{mm}^{-3}$ with $35 \%$ lymphocytes (CD4/CD8 ratio of 0.4 ) and $7 \%$ neutrophils. Results from microbiological testing were negative (including staining and culture for bacteria,

$@$ ERSpublications

Three cases of pembrolizumab-induced pneumonitis are described, two being consistent with organising pneumonia http://ow.ly/Dvhc308QI39

Cite this article as: Leroy V, Templier C, Faivre J-B, et al. Pembrolizumab-induced pneumonitis. ERJ Open Res 2017; 3: 00081-2016 [https://doi.org/10.1183/23120541.00081-2016]. 


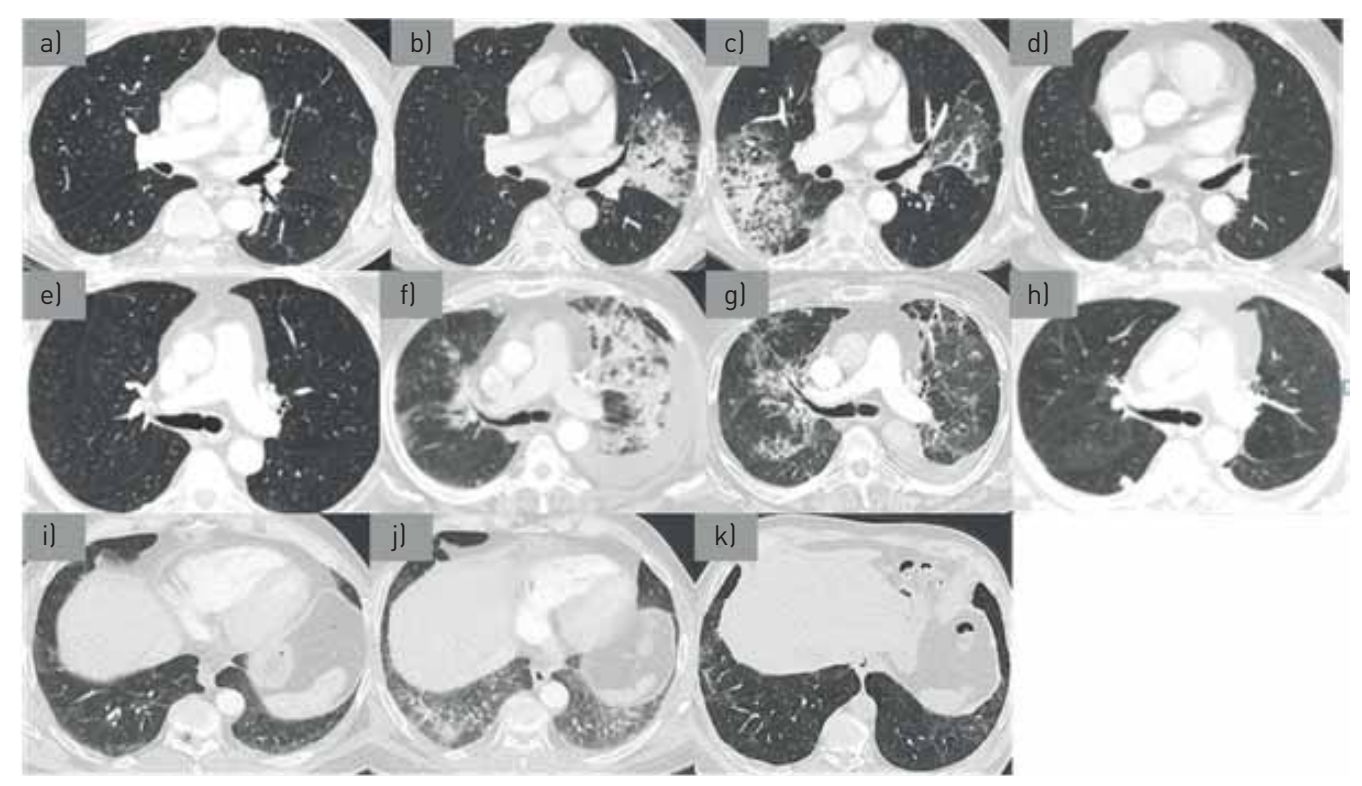

FIGURE 1 a) CT scan of patient number 1, before pembrolizumab; b) CT scan of patient number 1, after 11 pembrolizumab infusions; c) CT scan of patient number 1, after empiric antibiotics; d) CT scan of patient number 1, 3 months after corticosteroids were started; e) CT scan of patient number 2 before pembrolizumab; f) CT scan of patient number 2, after 4 pembrolizumab infusions; gl CT scan of patient number 2,1 month after pembrolizumab was stopped; h) CT scan of patient number 2, 2 months after corticosteroids were started; i. CT scan of patient number 3 , before pembrolizumab; j) CT scan of patient number 3 , after 4 pembrolizumab infusions; k) CT scan of patient number 3, 2 months after pembrolizumab was stopped.

fungi, viruses, mycobacteria and Pneumocystis jirovecii). The patient had restrictive ventilatory disorder with a forced expiratory volume in $1 \mathrm{~s}$ of $40 \%$ predicted, forced vital capacity of $47 \%$ predicted and a total lung capacity of $78 \%$. A minimum arterial oxygen saturation measured by pulse oximetry $\left(\mathrm{SpO}_{2}\right)$ of $85 \%$ was recorded in the 6-min walk test for a distance of $240 \mathrm{~m}$ covered. Results from complete laboratory tests were normal. Treatment with pembrolizumab was suspended. A new CT scan performed a month later revealed a decrease in the size and density of pre-existing lesions but the appearance of new consolidations (figure 1g). This spontaneous migration was suggestive of OP secondary to pembrolizumab treatment. Oral prednisolone was started at $0.5 \mathrm{mg}^{-1} \cdot \mathrm{kg}^{-1} \cdot \mathrm{day}^{-1}$ for 12 weeks and then slowly tapered for an expected 52 weeks of treatment. The patient's clinical and radiological symptoms improved; however, multiple lung metastasis appeared on the follow-up CT scan (figure 1h). The patient had multiple metastatic progression and died at 4 months without treatment.

The third patient was a 64-year-old man who had received a diagnosis of melanoma 2 years earlier. He was initially treated with local resection, lymphadenopathy, interferon therapy and radiation therapy. The disease progressed with pleural metastasis and pembrolizumab treatment $\left(2 \mathrm{mg}^{-1} \cdot \mathrm{kg}^{-1}\right.$ every 2 weeks $)$ was begun. A CT scan performed before pembrolizumab initiation revealed minimal reticulations and ground-glass opacities in the sub-pleural regions of the lower lobes (figure 1i). After four infusions, a repeat CT scan showed increased ground-glass infiltrates and reticulations indicative of a non-specific interstitial pneumonia (NSIP) pattern (figure $1 \mathrm{j}$ ). The patient was asymptomatic and pulmonary function tests were not performed. Pembrolizumab treatment was suspended and no other treatment was given. Two months later, a new CT scan showed regression of the ground-glass opacities and sub-pleural reticulation in the lower lobe. However, bronchiolectasis appeared superimposed on the infiltrates (figure $1 \mathrm{k}$ ). For oncological reasons, pembrolizumab was cautiously reintroduced. After rechallenge (two infusions), a CT scan showed stable disease. Narrow follow-up is ongoing.

Pneumonitis due to anti-PD-1 is a known complication; however, few cases have been reported in the literature and no consensus has yet been obtained for the management of this adverse events. We here describe pneumonitis in a more accurate way to help clinicians recognise the symptoms. We determined that pneumonitis was associated with patterns of ILD, although no histological evidence was found. In our three cases, the patients were too weak for surgical lung biopsy and we considered the clinico-radiological pattern to be suggestive enough. Transbronchial cryobiopsies could also have helped but this procedure was not yet available in our centre. Based on radiological patterns, cases 1 and 2 were classified as OP and case 3 as NSIP. Nishino et al. [12] described three cases of ILD occurring during nivolumab treatment: two had acute interstitial pneumonia with acute respiratory distress syndrome and one had NSIP. 
More recently, a case of OP was described in a melanoma patient treated with nivolumab [11]. Only two cases of pulmonary side effects with pembrolizumab have thus far been described: one case of OP occurring after four infusions and one case of mediastinal granulomatosis [13-15]. In clinical trials of pembrolizumab and nivolumab, the majority of pneumonitis cases have been reported as mild to moderate in severity, but one death has occurred $[2-4,10]$. Our three patients were managed as outpatients and the patient with NSIP did not require treatment.

Little is known about the factors promoting pneumonitis in patients treated with anti-PD-1 antibodies, but it does not appear to be linked to the antibody dose. In previous trials, pembrolizumab-related pneumonitis was less frequent in melanoma patients $(<1 \%)$ than in non-squamous cell lung cancer patients $(\sim 5 \%)[2,3,10]$. Pneumonitis occurred more frequently in patients with a history of asthma/chronic obstructive pulmonary disease than in those without $(5.4 \%$ versus $3.1 \%)$ and more frequently in patients with a history of prior thoracic radiation than in patients without (6.0\% versus $2.6 \%)$ [16]. It is therefore quite interesting to note that all of our three patients had lung metastasis. In our third case, NSIP appeared in a patient with underlying minimal ILD. Thus, triggering of pneumonitis might be facilitated by pre-existing local inflammation. Immune-related adverse events are usually treated with immunomodulatory medications, with steroids as the first line of treatment [17]. It is not yet known how long-term steroid therapy affects the disease course or treatment efficiency. Immune checkpoint inhibitors are very promising anti-tumour treatments but require dedicated management, including early diagnosis and treatment of immune-related adverse events.

Vincent Leroy ${ }^{1}$, Carole Templier ${ }^{2}$, Jean-Baptiste Faivre ${ }^{3}$, Arnaud Scherpereel ${ }^{4,5}$, Clement Fournier ${ }^{6}$, Laurent Mortier $^{2,7}$ and Lidwine Wemeau-Stervinou ${ }^{1}$

${ }^{1}$ Service de Pneumologie et Immuno-allergologie, Centre de Compétences des Maladies Pulmonaires Rares, CHU Lille, Lille, France. ${ }^{2}$ Service de Dermatologie, CHU Lille, Lille, France. ${ }^{3}$ Service de Radiologie et Imagerie Thoracique, CHU Lille, Lille, France. ${ }^{4}$ Service de Pneumologie et Oncologie Thoracique, CHU Lille, Lille, France. ${ }^{5}$ Institut Pasteur de Lille, U1019-CIIL-Center for Infection and Immunity of Lille, Université de Lille, Inserm, Lille, France. ${ }^{6}$ Service d’Endoscopie Respiratoire, CHU Lille, Lille, France. ${ }^{7}$ U1189-ONCO-THAI, University de Lille, Inserm, Lille, France.

Correspondence: Lidwine Wemeau-Stervinou, Service de Pneumologie et Immuno-allergologie, Centre de Compétences des Maladies Pulmonaires Rares, Hôpital Calmette, Boulevard du Pr Jules Leclercq, 59037 Lille, France. E-mail: lidwine.wemeau@chru-lille.fr

Received: July 172016 | Accepted after revision: Jan 192017

Conflict of interest: Disclosures can be found alongside this article at openres.ersjournals.com

\section{References}

1 Tumeh PC, Harview CL, Yearley JH, et al. PD-1 blockade induces responses by inhibiting adaptive immune resistance. Nature 2014; 515: 568-571.

2 Garon EB, Rizvi NA, Hui R, et al. Pembrolizumab for the treatment of non-small-cell lung cancer. $N$ Engl $J$ Med 2015; 372: 2018-2028.

3 Robert C, Schachter J, Long GV, et al. Pembrolizumab versus ipilimumab in advanced melanoma. N Engl J Med 2015; 372: 2521-2532.

4 Ribas A, Puzanov I, Dummer R, et al. Pembrolizumab versus investigator-choice chemotherapy for ipilimumab-refractory melanoma (KEYNOTE-002): a randomised, controlled, phase 2 trial. Lancet Oncol 2015; 16: 908-918.

5 Borghaei H, Paz-Ares L, Horn L, et al. Nivolumab versus docetaxel in advanced nonsquamous non-small-cell lung cancer. N Engl J Med 2015; 373: 1627-1639.

6 Weber JS, D'Angelo SP, Minor D, et al. Nivolumab versus chemotherapy in patients with advanced melanoma who progressed after anti-CTLA-4 treatment (CheckMate 037): a randomised, controlled, open-label, phase 3 trial. Lancet Oncol 2015; 16: 375-384.

7 Robert C, Long GV, Brady B, et al. Nivolumab in previously untreated melanoma without BRAF mutation. $N$ Engl J Med 2015; 372: 320-330.

8 Rizvi NA, Mazières J, Planchard D, et al. Activity and safety of nivolumab, an anti-PD-1 immune checkpoint inhibitor, for patients with advanced, refractory squamous non-small-cell lung cancer (CheckMate 063): a phase 2, single-arm trial. Lancet Oncol 2015; 16: 257-265.

9 Gettinger SN, Horn L, Gandhi L, et al. Overall survival and long-term safety of nivolumab (anti-programmed death 1 antibody, BMS-936558, ONO-4538) in patients with previously treated advanced non-small-cell lung cancer. J Clin Oncol 2015; 33: 2004-2012.

10 Herbst RS, Baas P, Kim D-W, et al. Pembrolizumab versus docetaxel for previously treated, PD-L1-positive, advanced non-small-cell lung cancer (KEYNOTE-010): a randomised controlled trial. Lancet 2015; 387: 1540-1550.

11 Sano T, Uhara H, Mikoshiba Y, et al. Nivolumab-induced organizing pneumonia in a melanoma patient. Jpn J Clin Oncol 2016; 46: 270-272.

12 Nishino M, Sholl LM, Hodi FS, et al. Anti-PD-1-related pneumonitis during cancer immunotherapy. $N$ Engl J Med 2015; 373: 288-290. 
13 Fragkou P, Souli M, Theochari M, et al. A case of organizing pneumonia (OP) associated with pembrolizumab. Drug Target Insights 2016; 10: 9-12.

14 Cousin S, Toulmonde M, Kind M, et al. Pulmonary sarcoidosis induced by the anti-PD1 monoclonal antibody pembrolizumab. Ann Oncol 2016; 27: 1178-1179.

15 Paydas S. Pulmonary sarcoidosis induced by the anti-PD-1 monoclonal antibody pembrolizumab or post-immunotherapy granulomatous reaction: which is more appropriate terminology? Ann Oncol 2016; 27: $1650-1651$.

16 Sul J, Blumenthal GM, Jiang X, et al. FDA approval summary: pembrolizumab for the treatment of patients with metastatic non-small cell lung cancer whose tumors express programmed death-ligand 1. Oncologist 2016; 21 : 643-650.

17 Spain L, Diem S, Larkin J. Management of toxicities of immune checkpoint inhibitors. Cancer Treat Rev 2016; 44 51-60. 